\title{
Evaluations of the deuteron induced reactions by using a semi-microscopic approach based on the CDCC-DWBA formalism
}

Pierre Chau Huu-Tai ${ }^{\mathrm{a}}$

CEA, DAM, DIF, 91297 Arpajon, France

\begin{abstract}
We propose to use the Continuum Discretized Coupled Channels (CDCC) formalism to build some evaluations of deuteron induced reactions. In particular a semi-microscopic model based upon the CDCCDWBA framework is used to compute the $(\mathrm{d}, \mathrm{p})$ cross sections. We have then built a functional to reproduce these calculations and we have included it into Talys. As an illustration, some excitation functions for $\mathrm{d}+\mathrm{Ni}$ reactions have been compared with the experimental data: a good agreement between experimental cross sections and the calculated ones is then obtained.
\end{abstract}

\section{Introduction}

Deuteron induced reactions are relevant to produce some isotopes usefull for medical or material applications. Therefore it seems important to propose accurate libraries of evaluated cross sections for these reactions. A promising way to build such libraries is to use the Continuum Discretized Coupled Channels approach. Indeed, this formalism which was proposed in 1974 by G.W. Rawitscher [1] explicitly includes the elastic breakup channels into the calculations of the cross sections. Since then this approach has been widely studied and quite sucessfully used for this kind of reaction [2-7]. This formalism has been extended to include the target excitations (see e.g., [12]) and also to investigate reactions involving weakly bound projectiles such as $\mathrm{Be}$ and $\mathrm{Li}$ isotopes: some core excitations have been included in [8] and the scattering of four-body systems has been studied e.g., in [9-11]. We thus propose to use it to compute $\mathrm{d}$ induced reactions. Moreover, since a large part of the $(\mathrm{d}, \mathrm{p})$ reaction is expected to come from direct interaction, we propose to build a semi-microscopic model relying on CDCC-DWBA calculations to get the excitation function of this transfer reaction. In the next section, we very briefly summarize the approach that has been used to estimate the $(d, p)$ excitation function. The third section is devoted to the comparison between the calculations and the experimental cross sections for $\mathrm{Ni}$ isotopes. We end by some remarks and conclusions.

\section{Computation of the (d,p) excitation function using a functional deduced from a semi-microscopic model}

\subsection{A semi-microscopic model for the $(d, p)$ excitation function}

To compute the $(\mathrm{d}, \mathrm{p})$ excitation function we use the semimicroscopic model which is decribed in more detail in [12]

\footnotetext{
a e-mail: huu-tai.chau@cea.fr
}

and which is briefly recalled here for self-consistency. The cross section of any reaction at a given incident energy, $E_{d}$, can be written as the summation of three contributions:

$$
\sigma\left(E_{d}\right)=\sigma_{\text {Direct }}\left(E_{d}\right)+\sigma_{\mathrm{CN}}\left(E_{d}\right)+\sigma_{\text {Pre-eq. }}\left(E_{d}\right)
$$

where $\sigma_{\text {Direct }}, \sigma_{\mathrm{CN}}$ and $\sigma_{\text {Pre-eq. }}$ denote the direct component, the Compound Nucleus and the preequilibrium one, respectively. For the $(\mathrm{d}, \mathrm{n})$ or $(\mathrm{d}, \mathrm{p})$ cross section, the direct component can be expressed as

$$
\sigma_{\text {Direct }}^{(d, \text { nucleon })}\left(E_{d}\right)=\sum_{i \leq i_{\max }, l_{n}, j_{n}} \sigma_{l_{n}, j_{n}}\left(E_{x, i}, E_{d}\right)
$$

where $\sigma_{l_{n}, j_{n}}\left(E_{x, i}, E_{d}\right)$ is the cross section when the nucleon is captured by the target to form the $\mathrm{A}+1$ nucleus into a state whose orbital angular momentum, spin and excitation energy, are $l_{n}, j_{n}, E_{x, i}$, respectively, $i_{\max }$ depending on the incident energy and the Q-value of the reaction. Therefore to obtain this direct component, one should firstly determine all the states that can be populated and then for each state, one should compute the associated cross section $\sigma_{l_{n}, j_{n}}\left(E_{x, i}, E_{d}\right)$ which can be obtained by integrating the differential cross section:

$$
\sigma_{l_{n}, j_{n}}\left(E_{x, i}, E_{d}\right)=\int \frac{d \sigma_{l_{n}, j_{n}}}{d \Omega}\left(E_{x, i}, E_{d}\right) d \Omega .
$$

It is usually assumed that the level with excitation energy $E_{x, i}$ is a pure single-particle state which can be deduced by using a Woods-Saxon potential whose depth is adjusted to get the correct excitation energy. To take into account that the state is not in fact a pure single-particle one, a spectroscopic factor, $S_{l_{n}, j_{n}, i}$, is introduced to renormalize the calculated cross section, which means that, for each level, the experimental cross section must be known for at least one incident energy:

$$
\begin{aligned}
& \sigma_{l_{n}, j_{n}}\left(E_{x, i}, E_{d}\right)=S_{l_{n}, j_{n}, i} \int \frac{d \sigma_{l_{n}, j_{n}}^{\text {calc. }}}{d \Omega}\left(E_{x, i}, E_{d}\right) d \Omega \\
& \Rightarrow \sigma_{\text {Direct }}^{(d, \text { nucleon })}\left(E_{d}\right)=\sum_{i \leq i_{\max }, l_{n}, j_{n}} S_{l_{n}, j_{n}, i} \int \frac{d \sigma_{l_{n}, j_{n}}^{\text {calc. }}}{d \Omega}\left(E_{x, i}, E_{d}\right) d \Omega .
\end{aligned}
$$


However with such an approach, one faces several drawbacks. Indeed the determination of the populated levels is far from being trivial and it is often impossible to get an accurate knowledge of the spectroscopic factors. That is why we have adopted a simplified approach relying on the fact that we have numerically observed [12] that the shape of the excitation function of the integrated cross sections does not depend strongly on the level excitation energy but mainly on the orbital angular momentum. Thus we will assume that:

$$
\sigma_{l_{n}, j_{n}}^{\text {calc. }}\left(E_{x, i}, E_{d}\right) \propto \tilde{\sigma}_{l_{n}, j_{n}}\left(E_{d}\right),
$$

meaning that the integrated cross sections can now be written as the product of an amplitude depending on the excitation energy of the level, $A\left(E_{x, i}\right)$, times a function depending only on $l_{n}$ and on the incident energy:

$$
\sigma_{l_{n}, j_{n}}^{\text {calc. }}\left(E_{x, i}, E_{d}\right) \approx A\left(E_{x, i}\right) \tilde{\sigma}_{l_{n}, j_{n}}\left(E_{d}\right) .
$$

If for each $l_{n}$, a level with a given excitation energy $E_{x, i_{0}}$ is chosen and the associated cross section $\sigma_{l_{n}, j_{n}, i_{0}}^{\text {calc }}\left(E_{d}\right)$ is computed then:

$$
\begin{aligned}
& \sigma_{l_{n}, j_{n}, i_{0}}^{\text {cdcc-dwba }}\left(E_{d}\right) \equiv \sigma_{l_{n}, j_{n}}^{\text {calc. }}\left(E_{x, i_{0}}, E_{d}\right)=A\left(E_{x, i_{0}}\right) \tilde{\sigma}_{l_{n}, j_{n}}\left(E_{d}\right) \\
& \Rightarrow \forall E_{x, i}, \sigma_{l_{n}, j_{n}}^{\text {calc. }}\left(E_{x, i}, E_{d}\right) \approx \frac{A\left(E_{x, i}\right)}{A\left(E_{x, i_{0}}\right)} \sigma_{l_{n}, j_{n}, i_{0}}^{\text {cdcc-dwba }}\left(E_{d}\right) .
\end{aligned}
$$

The direct component of excitation function can now be transformed as follows:

$$
\begin{aligned}
\sigma_{\text {Direct }}^{(d, \text { nucleon })}\left(E_{d}\right) & =\sum_{i \leq i_{\max }, l_{n}, j_{n}} \sigma_{l_{n}, j_{n}}\left(E_{x, i}, E_{d}\right) \\
& \approx \sum_{l_{n}, j_{n}}\left(\sum_{i \leq i_{\max }} S_{l_{n}, j_{n}, i} \frac{A\left(E_{x, i}\right)}{A\left(E_{x, i_{0}}\right)}\right) \sigma_{l_{n}, j_{n}, i_{0}}^{\text {cdcc-dwba }}\left(E_{d}\right) .
\end{aligned}
$$

Denoting $\sum_{i \leq i_{\max }} S_{l_{n}, j_{n}, i} \frac{A\left(E_{x, i}\right)}{A\left(E_{x, i_{0}}\right)}=W_{l_{n}, j_{n}}\left(E_{d}\right)$, the direct contribution can be rewritten as:

$$
\sigma_{\text {Direct }}^{(d, \text { nucleon })}\left(E_{d}\right) \approx \sum_{l_{n}, j_{n}} W_{l_{n}, j_{n}}\left(E_{d}\right) \sigma_{l_{n}, j_{n}, i_{0}}^{\text {cdcc-dwba }}\left(E_{d}\right)
$$

where the weights $W_{l_{n}, j_{n}}$ 's are non-decreasing functions of $E_{d}$. Moreover, since the number of states is finite, it is deduced that

$$
\begin{aligned}
\lim _{E_{d} \rightarrow \infty} & W_{l_{n}, j_{n}}\left(E_{d}\right)=\omega_{l_{n}, j_{n}} \\
& \Rightarrow \sigma_{\text {Direct }}^{(d, \text { nucleon })}\left(E_{d}\right) \widetilde{\infty} \sum_{l_{n}, j_{n}} \omega_{l_{n}, j_{n}} \sigma_{l_{n}, j_{n}, i_{0}}^{\text {cdcc-dwba }}\left(E_{d}\right),
\end{aligned}
$$

meaning that the $(\mathrm{d}, \mathrm{p})$ excitation function can be approximated by a linear combination of the individual cross sections, $\sigma_{l_{n}, j_{n}, i_{0}}^{\text {cdcc-dwa }}$. The weights can be determined by using a very limited number of experimental data: since the main contributions seem to come from $l_{n}=0, l_{n}=1$ and $l_{n}=2$, the values of the excitation function at less than three incident energies are needed to find these weights.

To compute the $\sigma_{l_{n}, j_{n}, i_{0}}^{\text {cdcc-dwba }}$ cross section, the CDCCDWBA approach has been used: the elastic and breakup channels are computed within the CDCC aproach then the transfer cross section is determined. For the CDCC calculations, for the $S$ and $D$ waves, the continuum

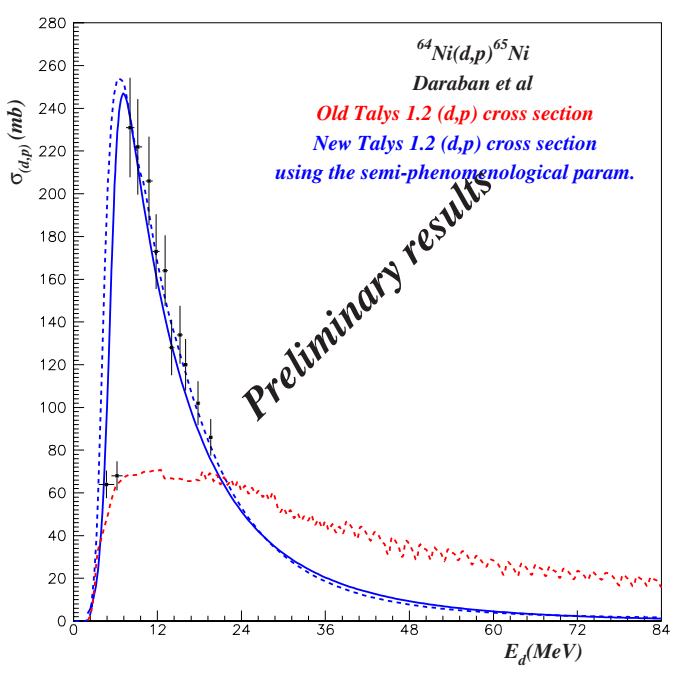

Figure 1. Comparison of the Talys cross sections ${ }^{64} \mathrm{Ni}(\mathrm{d}, \mathrm{p}){ }^{65} \mathrm{Ni}$ with the experimental ones [18-21].

discretization has been performed by using 4 bins. We have used the Koning-Delaroche paramaterization [13] for the nucleon-target optical potentials, a Gaussian potential for the p-n interaction and a Woods-Saxon potential to get the neutron wave function. We call the excitation function given by (6) a semi-microscopic excitation function since it relies on a "microscopic" one, $\sigma_{l_{n}, j_{n}, i_{0}}^{\text {cdcc-d }}$, and on the phenomenological weight $\omega_{l_{n}, j_{n}}$ (we could have called it a semi-phenomenological excitation function).

\subsection{A fit of the semi-microscopic (d,p) excitation function}

And finally since we wish to use a reaction code like Talys and since the full CDCC-DWBA computations are quite time consuming, we have proposed to fit our computations by some functional (during the conference, the author has been informed by B.V. Carlson that A.V. Ignatyuk has proposed the same kind of functional built upon a systematic study of the experimental data [17]) and to include it into a new subroutine of Talys. Thus we compute the CDCC-DWBA excitation functions for 78 nuclei and then we fit each one by the following functional:

$$
\begin{aligned}
\sigma_{(d, p)}^{F i t}\left(E_{d}\right)= & \sigma_{0}\left(1-\frac{1}{1+\exp \left(\frac{E_{d}-E_{0}}{\Delta E_{0}}\right)}\right) \\
& \times\left(A_{1} \exp \left(-\lambda_{1} E_{d}\right)+A_{2} \exp \left(-\lambda_{2} E_{d}\right)\right) .
\end{aligned}
$$

The trends of the parameters of $\sigma_{(d, p)}^{F i t}$ as function of the neutron number have been studied and some shell effects have been observed and presented during the conference.

\section{Comparison with some $\mathrm{d}+\mathrm{Ni}$ experimental cross sections}

The comparisons of our calculations with the experimental cross sections [18-21] are illustrated in Figs. 1-6.

\section{Conclusion}

Building accurate nuclear data libraries for deuteron induced reaction is of importance for many fields such 


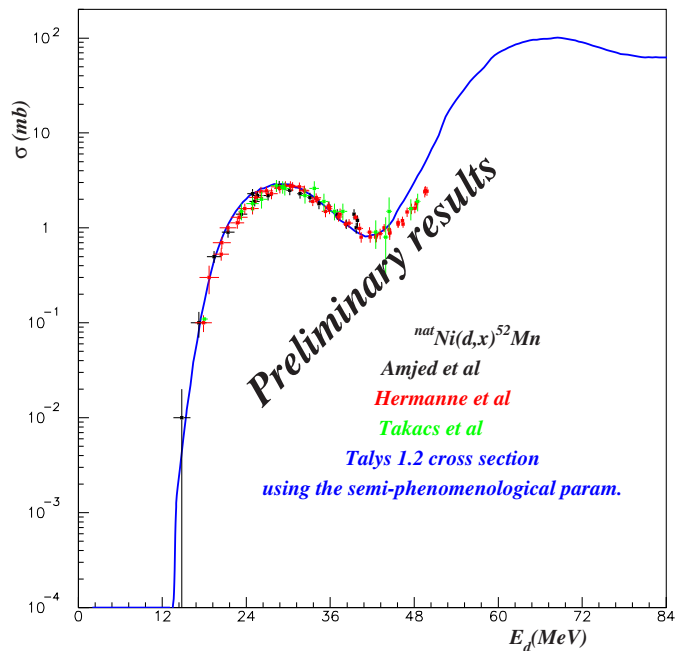

Figure 2. Comparison of the Talys cross sections ${ }^{n a t} \mathrm{Ni}(\mathrm{d}, \mathrm{x})^{52} \mathrm{Mn}$ with with the experimental ones [18-21].

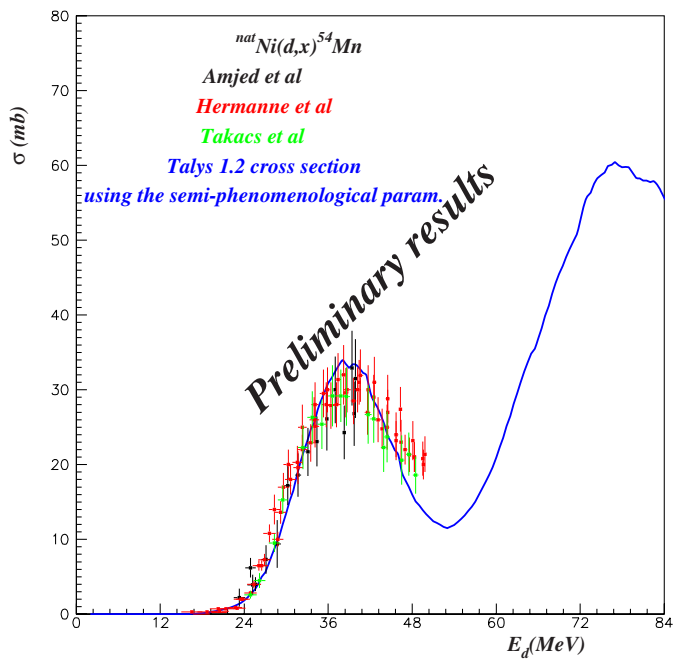

Figure 3. Comparison of the Talys cross sections ${ }^{\text {nat }} \mathrm{Ni}(\mathrm{d}, \mathrm{x})^{54} \mathrm{Mn}$ with with the experimental ones [18-21].

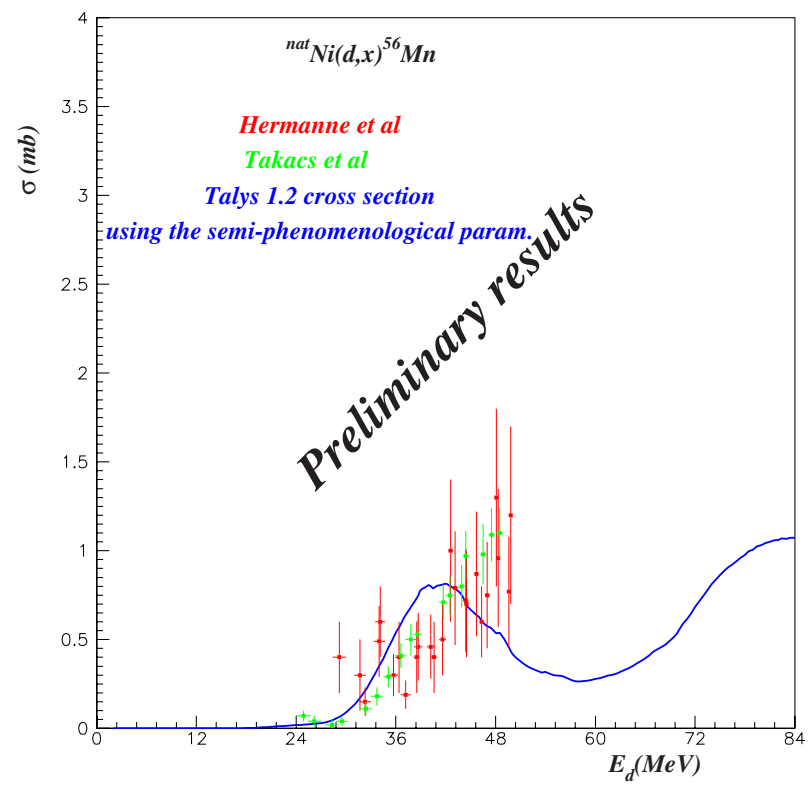

Figure 4. Comparison of the Talys cross sections ${ }^{n a t} \mathrm{Ni}(\mathrm{d}, \mathrm{x})^{56} \mathrm{Mn}$ with with the experimental ones [18-21].

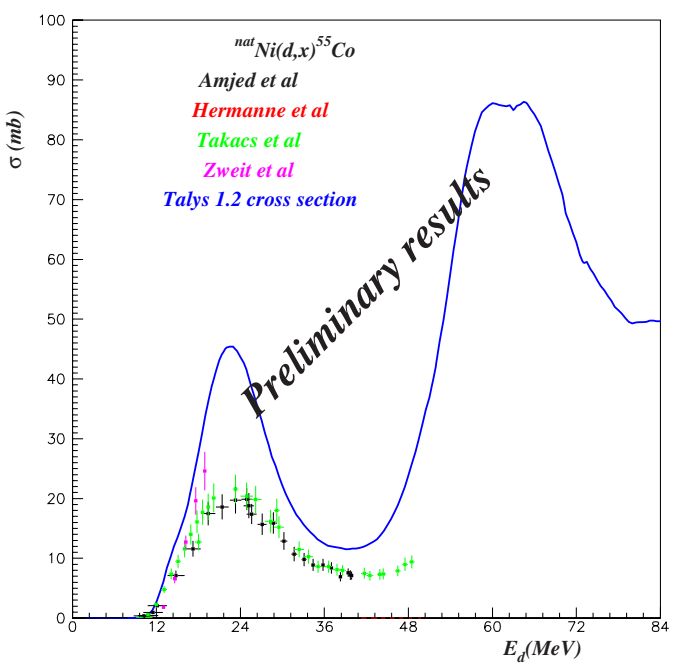

Figure 5. Comparison of the Talys cross sections ${ }^{n a t} \mathrm{Ni}(\mathrm{d}, \mathrm{x})^{55} \mathrm{Co}$ with the experimental ones [19-21].

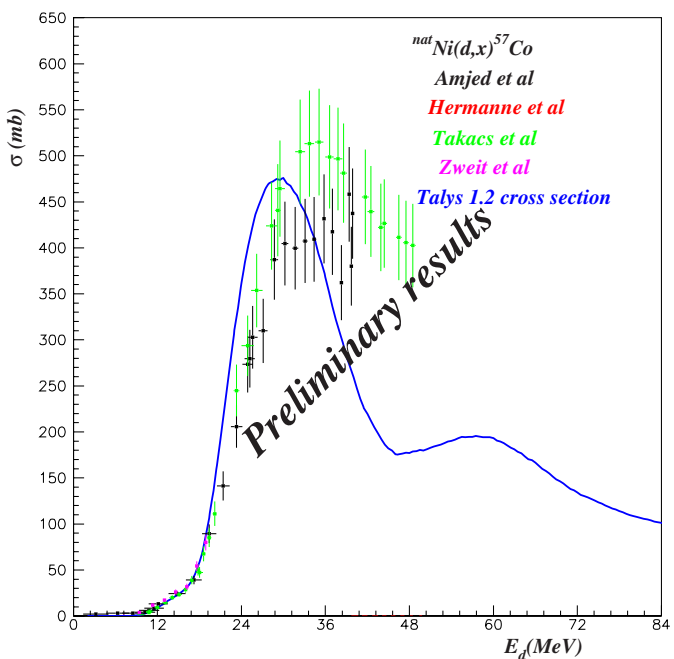

Figure 6. Comparison of the Talys cross sections ${ }^{\text {nat }} \mathrm{Ni}(\mathrm{d}, \mathrm{x})^{57} \mathrm{Co}$ with the experimental ones [19-21].

as the production of radio-isotopes relevant for medical applications or the evaluation of irradiation damage of fusion materials and recently, many theoretical efforts have been performed e.g., by S. Nakayama et al. [22-24] and by P. Bém et al. [25] to improve these calculations. We have also produced some evaluations for $\mathrm{d}+\mathrm{Ni}$ isotopes using Talys combined with calculations based on the CDCCDWBA approach to get the direct component of the $(\mathrm{d}, \mathrm{p})$ excitation function and a quite good agreement between our computations and the experimental data has been obtained. We hope that the accuracy of the excitations for these reactions will be soon comparable to the one obtained for neutron induced reactions.

\section{References}

[1] G.H. Rawitscher, Phys. Rev. C 9, 2210 (1974)

[2] M. Yahiro and M. Kamimura, Prog. Theor. Phys. 65 2046 (1981)

[3] M. Yahiro and M. Kamimura, Prog. Theor. Phys. 65, 2051 (1981)

[4] M. Yahiro et al., Prog. Theor. Phys. 67, 1467 (1982) 
[5] M. Yahiro et al., Prog. Theor. Phys. Suppl. 89, 1 (1986)

[6] N. Austern et al., Phys. Rep. 154, 125 (1987)

[7] P. Chau-Huu Tai, Nucl. Phys. A 773, 56 (2006)

[8] N.C. Summers et al., Phys. Rev. C 76, 014611 (2007)

[9] T. Matsumoto et al., Phys. Rev. C 70, 061601 (2004)

[10] M. Rodríguez-Gallardo et al., Phys. Rev. C 80, 051601 (2009)

[11] P.N. de Faria et al., Phys. Rev. C 81, 044605 (2010)

[12] P. Chau Huu-Tai, Eur. Phys. J. A 51, 166 (2015)

[13] A.J. Koning and J.P. Delaroche, Nucl. Phys. A 713, 231 (2003)

[14] M. Masaki et al., Nucl. Phys. A 573, 1 (1994)

[15] K. Hirota et al., Nucl. Phys. A 628, 547 (1998)

[16] M. Yamaguchi et al., Nucl. Phys. A 747, 3 (2005)
[17] A.V. Ignatyuk, unpublished (2011). Pheno menological systematics of the $(\mathrm{d}, \mathrm{p})$ cross sections. http://www-nds.iaea.org/fendl3/ 000pages/rcm3/slides// ignatyukfendl-3

[18] L. Daraban et al., Appl. Rad. Iso. 67, 506 (2009)

[19] N. Amjed et al., Appl. Rad. Iso. 82, 87 (2013)

[20] A. Hermanne et al., Nucl. Inst. Meth. B 299, 8 (2013)

[21] S. Takács et al., , Nucl. Inst. Meth. B 260, 495 (2007)

[22] S. Nakayama et al., Energy Procedia 71, 219 (2015)

[23] S. Nakayama et al., Phys. Rev. C 94, 014618 (2016)

[24] S. Nakayama and Y. Watanabe, J. Nucl. Science and Technology 53, 89 (2016)

[25] P. Bém et al., Phys. Rev. C 79, 044610 (2009) 